\title{
Severe Flexion Contracture of the Finger of a 16 Years Old Boy
}

\author{
${ }^{*}$ Haque $M A^{1}$, Haque $\mathrm{ME}^{2}$.
}

\begin{abstract}
Postburn flexion contractures of the finger are very common conditions presenting for treatment. Similarly trauma to the upper limbs results in flexion deformities of the fingers. Standard treatment for these deformities is by way of surgical release of the contractures, skin cover with split skin grafting or full thickness grafts followed by prolonged immobilization and splint age. Here we described a rare presentation of severe flexion contracture of the both proximal (PIP) and distal interphalangeal (DIP) joints of the right ring finger resulting from post traumatic infection of a 16 years old boy. The relatively minimally invasive technique of distraction has been used in this patient. Almost full range of movement of both PIP and DIP joints with strength of grip has returned after 12 weeks of operation. Result of treatment was excellent with no significant complication.
\end{abstract}

CBMJ 2012 July: Vol 01 No 02: P: 51-55

Key words: Flexion contracture, Release, Skin graft, Cross finger flap, Distractor.

\section{Introduction}

Flexion contracture of the both proximal (PIP) and distal interphalangeal (DIP) joints of the finger is less common than flexion contracture of proximal interphalangeal joint only. This type of flexion contracture significantly impairs hand function. The common causes are burn, trauma and infection. Several anatomical structures, including the skin, subcutaneous tissues, tendon sheath, flexor tendons, palmar plate, check rein ligament, and collateral ligaments are involved and shortened ${ }^{1-3}$. Various surgical techniques have been described with differing results for the treatment of flexion contractures of the PIP joints. The standard treatment of this contracture is release of all contracted tissue and resultant skin defect is covered by full thickness skin grafts or cross finger flap, prolonged immobilization and splintage. Recently, some researchers have achieved satisfactory results with the use of gradual distraction using an external fixator to correct traumatic flexion contracture ${ }^{4,5}$. Here we described a rare presentation of the severe flexion contracture of PIP and DIP joints of the ring finger of a 16 years old boy and its management and outcome.

\section{Case history}

A 16 years old boy presented in my chamber on 22.11.09 with history of trauma to the right ring finger 6 months back followed by abscess formation and incison and drainage was done by local doctor. Subsequently wound was healed and gradual bending of finger developed. Physical examination revealed flexion contracture of both PIP and DIP joints about 900 with few degrees of further flexion movements of both joints and extension. Examination revealed no evidence of neurovascular compromise in the finger. Other supportive investigations like X-ray, total and differential count of WBC, ESR, CRP excluded infection and involvement bony structure.

\footnotetext{
1. ${ }^{*}$ Dr. Md. Anwarul Haque

Associate Professor,

Department of Orthopaedic Surgery,

Community Based Medical College Hospital, Bangladesh.
}

2. Dr. Md. Emdadul Haque

Medical Officer, UHC, Trishal, Mymensingh

\footnotetext{
*Address of Correspondence:

E-mail: anwarul_dr@yahoo.com

Mobile: +8801711619314
} 


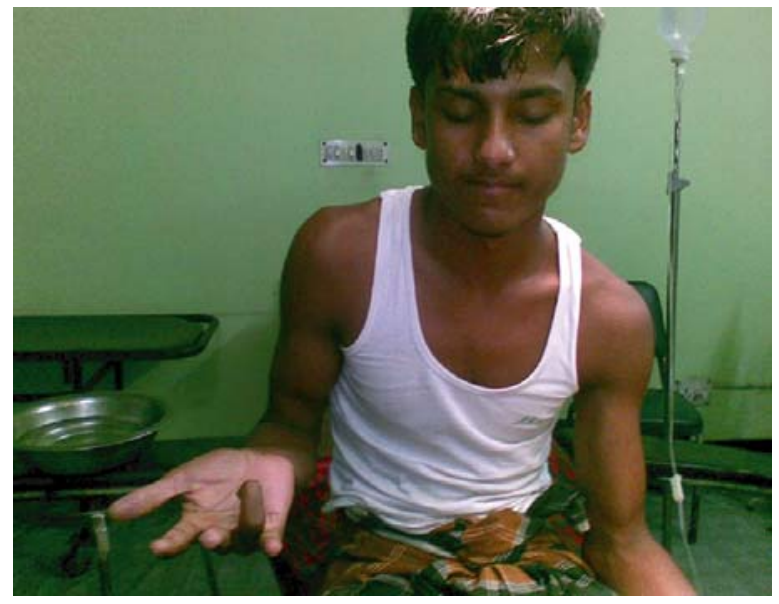

Preoperative Photograph

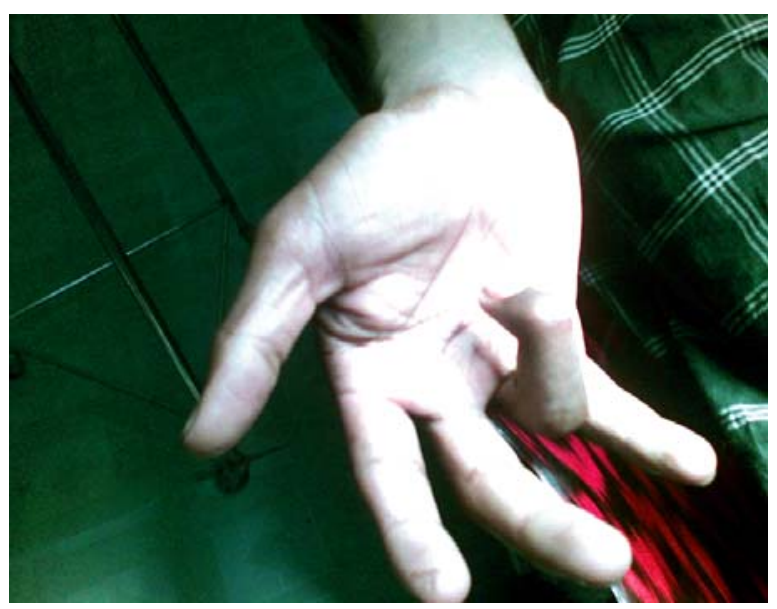

Preoperative Photograph

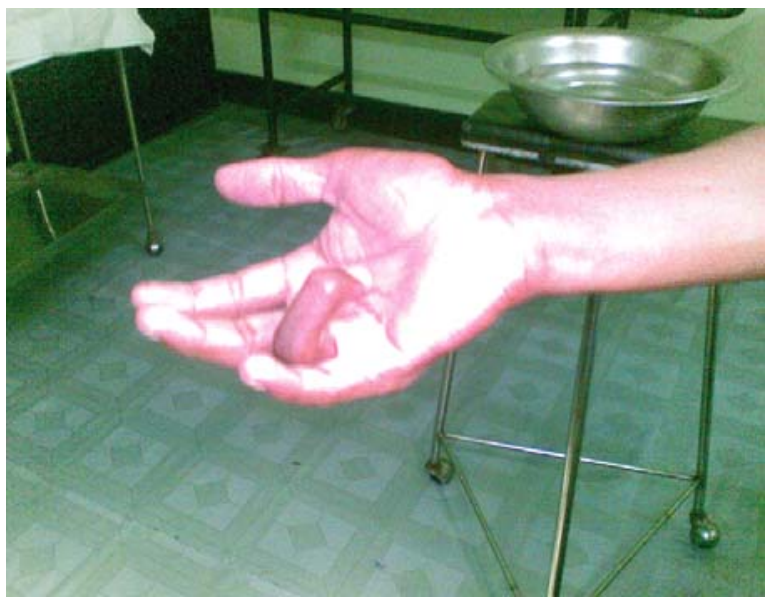

Preoperative Photograph

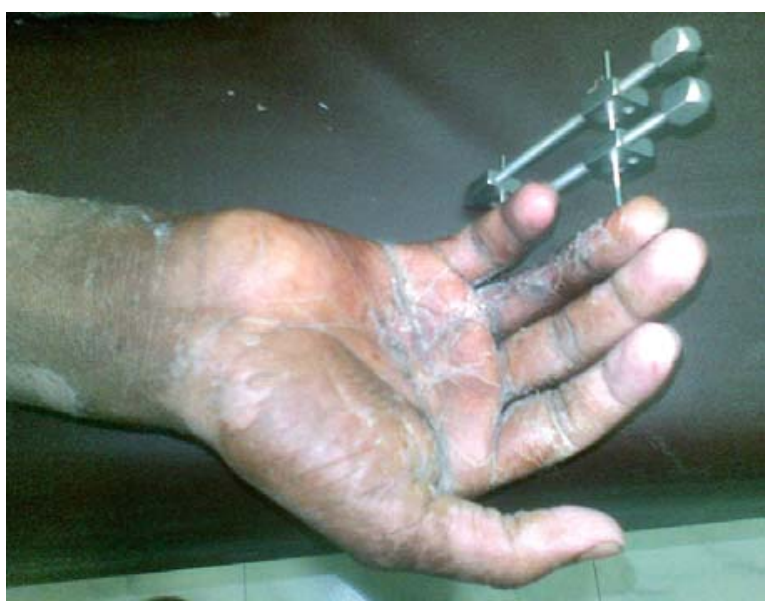

Photograph 6 weeks after operation

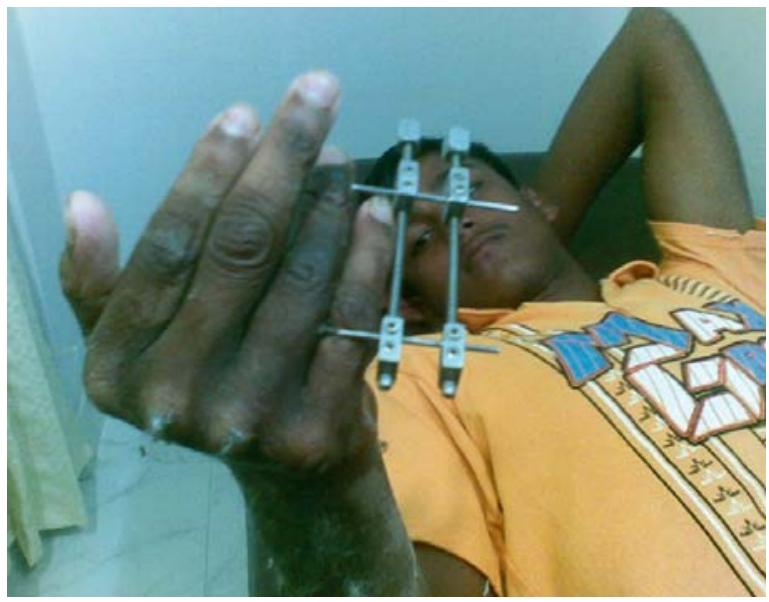

Photograph 6 weeks after operation 


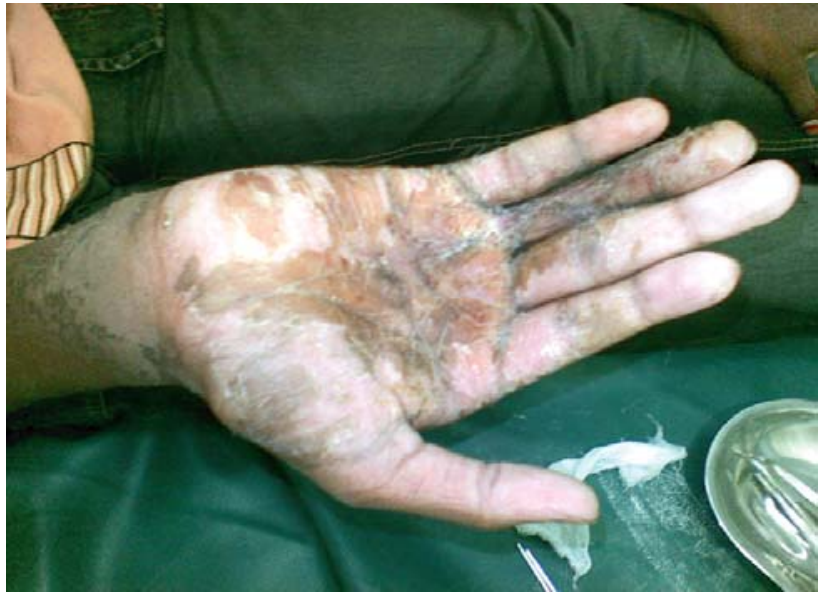

Photograph 10 weeks after operation with removed distractor

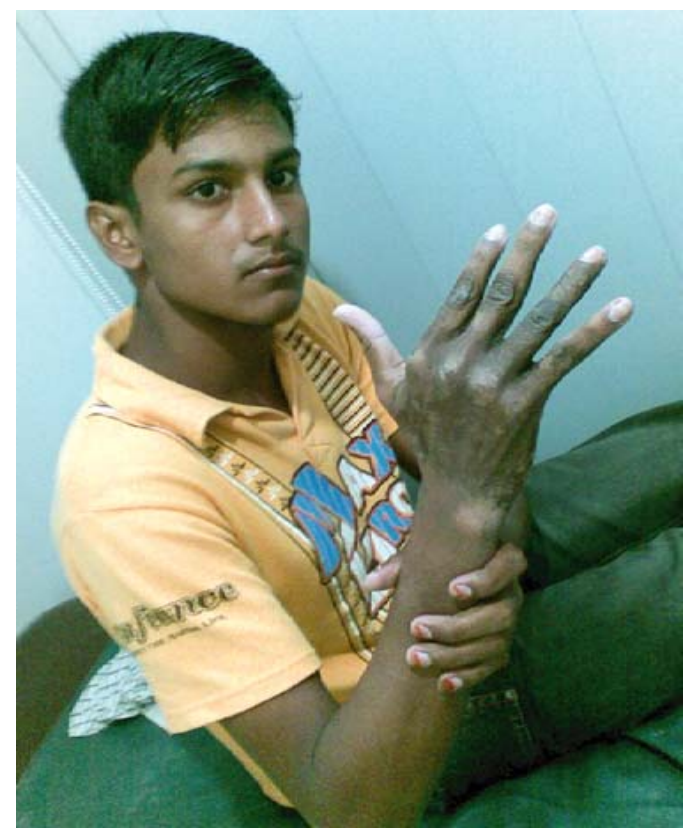

Photograph 12 weeks after operation

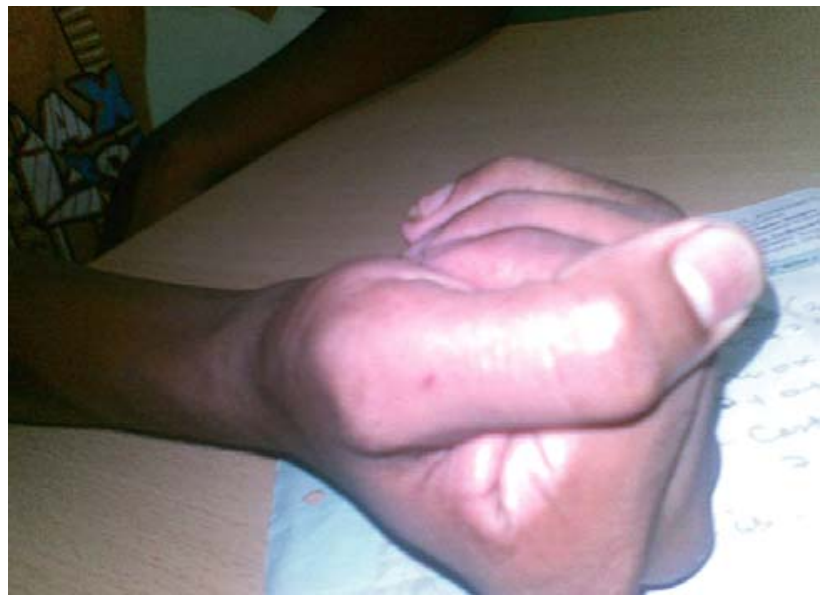

Photograph 12 weeks after operation

Oblique crease cross incision was given in the palmar surface of the finger. After incision skin margin is released from underlying contracted tissue. Then excision of the scar tissue and the contracted soft tissues under the skin were released sequentially from the check rein ligaments and palmar plates to collateral ligaments of the finger. Then closure of the skin was done. Then two shanz screws of 1.5 $\mathrm{mm}$ were used. One shanz screw was passed transversely through proximal phalanx and another one passed through the distal phalanx maintaining adequate distance from the joint to ensure that the distraction forces applied straightened the joint and did not subluxate it. Two pin distractors were used. Up to $15 \%$ $20 \%$ straightening of the joint could be achieved during the application and opening of the distractor on the operating table. Stitches were removed at 10th POD and gradual distraction was done at the rate $1 \mathrm{~mm}$ per day till the fingers got straightened out. Initial distraction was done by me. Then the patient was trained to do the distraction by themselves at home, coming at weekly interval for follow up. Once the finger was straighten distractors were kept in place for further 4 weeks before removal. Intermittent splintage with the finger in extension was maintained for further 4 weeks. 
The results in this patient who had presented for treatment for functional purposes were excellent. He went back to his job with an improvement in function and was very happy, as not only did the finger become straight \& active function also returned.

\section{Discussion}

Vallecular cysts are retention cysts of minor The standard treatment of flexion contractures of the finger is surgical release and skin cover with a full thickness grafts ${ }^{6-8}$. Other authors have found no significant differences between a thick split skin graft and a full thickness graft $^{9}$ Though most authors say thin split skin grafts should be avoided ${ }^{6-8}$ some say that split skin grafts from the non-weight bearing plantar aspect of the foot give the best cosmetic results ${ }^{10,11}$.

However standard method of release surgery under anaesthesia, usually general anaesthesia, with harvesting of full thickness grafts, which in a patient with extensive burns may occasionally be a problem due to lack of donor sites. There are inherent complications of surgery viz damage to the neorovascular bundles during surgery; finger tip ischaemia when a chronically contracted finger is acutely straightened out putting stretch on the neurovascular bundles; a contracted but uninjured tendon preventing the full straightening of the finger; graft loss; wound infections etc. Another important practical factor is the immobilization of the hand in bandages and splints, and multiple dressings when grafting has been done.

Several authors have described the results after surgical correction of disabling flexion contractures of the PIP joints of the fingers with variable rates of success. In 1976, Sprague ${ }^{12}$ reported $13^{\circ}$ ROM gains of flexion contractures of the PIP joints after palmar capsular release. However, the ROM deteriorated and became less than that of the preoperative status at the last follow-up. In 1979, Gould and Nicholson ${ }^{13}$ reported $12^{\circ}$ to $13^{\circ}$ improvements of motion of the joints after palmar capsulectomy, and suggested that more severe problems the patients had in the diagnosis, the less motion they gained at the last follow-up. Brüser, et al. ${ }^{14}$ reported $30^{\circ}$ and $50^{\circ}$ of improvement of motion after surgical release with 2 different approaches, a palmar and a midlateral incision. They recommended the midlateral incision because of less soft tissue trauma than that of the palmar incision, which made early mobilization possible.

As the majority of studies have reported disappointing outcomes with the open technique, other authors have been concerned about distraction histogenesis in the treatment of chronic flexion contractures of the PIP joints $4,5,15$. In 2002, Haushian, et al.5 reported a mean of $38^{\circ}$ of improvement of motion with a mean ROM of $42^{\circ}$ by dynamic extension correction technique using compass hinge external fixator. In 2004, Houshian and Schrøeder ${ }^{15}$ also reported good short-term results using a monolateral frame for distraction correction in 10 patients with chronic flexion contractures of the PIP joints. Houshian and Chikkamuniyappa4 reported an increase of the mean active ROM by $63^{\circ}$ and the mean active extension by $47^{\circ}$ after gradual distraction correction using an external fixator without serious complications. However, they excluded patients with contractures from burn or crushing in their study. Although the technique has satisfactory clinical outcomes, it has limitations in patients with severe flexion contractures of the PIP joints combined with scarring.

Though some authors have mentioned the use of distraction for the treatment of contractures ${ }^{16,17}$ there are no welldocumented series reported on the use of distraction for post burn contractures of the fingers.

By distraction straightening the principles of a gradual dynamic lengthening of the skin and soft tissues are applied. All tissues get stretched out thus maintaining a sensate pliable skin cover. Though all of the researchers using distractor without releasing any contracted tissue, but here we used distractor after releasing some contracted 
easily carried out in adults as an outpatient procedure the requirement being of only regional anaesthesia which is given by the surgeon himself. No hospitalization is required and the patient is trained to open the distractor by himself at home. The distractors are reutilisable as only the K-wires are expendable.

\section{Conclusion}

We concluded that gradual distraction by an external fixator may be safe and effective for the correction of chronic post-traumatic contractures of the PIP and DIP joints; however, similar studies on a larger group may be necessary before this technique could be recommended universally. In addition this procedure can be adopted as an outpatient procedure for adults, and under digital blocks anaesthesia. The functional and cosmetic results of this procedure are highly satisfactory. The aim of this paper is to offer distraction as an easy and economic technique for treating post burn/traumatic contractures of the fingers.

\section{References}

1. Curtis RM. Surgical restoration of motion in the stiff interphalangeal joints of the hand. Bull Hosp Joint Dis. 1970;31:1-6.

2. Diao E, Eaton RG. Total collateral ligament excision for contractures of the proximal interphalangeal joint. $J$ Hand Surg Am. 1993;18:395-402.

3. Lorea $P$, Medina Henriquez $J$, Navarro $R$, Legaillard $P$, Foucher $G$. Anterior tenoarthrolysis for severe flexion contracture of the fingers (the "TATA" operation): a review of 50 cases. J Hand Surg Eur Vol. 2007;32:224229.

4. Houshian S, Chikkamuniyappa C. Distraction correction of chronic flexion contractures of PIP joint: comparison between two distraction rates. J Hand Surg Am. 2007;32:651-656.

5. Houshian S, Gynning B, Schrøder HA. Chronic flexion contracture of proximal interphalangeal joint treated with the compass hinge external fixator. A consecutive series of 27 cases. $J$ Hand Surg Br. 2002;27:356-358.

6. Huartz TT, Lanon DC, Lewis SR. Burned Hands. Plast Reconstr Surg 1975;56:21.
7. Park PH, Evans EB, Lanon DC. Prevention and correction of deformity after severe burns. Surg Clin N Am 1978;58:1279.

8. Beasly RW. Secondary repair of burned hands. Clin Plast Surg 1981;8:141.

9. Alexander JW, MacMillan BG, Martel $L$. Surgical correction of post burn flexion contractures in children. Plast Reconstr Surg 1981;68:218.

10. Jackson IT, Brown GED. A method of treating chronic flexion contractures of fingers. $\mathrm{Br} J$ Plast Surg 1970;23:373.

11. Pensler JM, Stewart $R$, Lewis SR. Reconstruction of the burned palm- FTG vs. SSG- a long-term follow-up. Plast Reconstr Surg 1988;81:46.

12. Sprague BL. Proximal interphalangeal joint contractures and their treatment. J Trauma. 1976;16:259-265.

13. Gould JS, Nicholson BG. Capsulectomy of the metacarpophalangeal and proximal interphalangeal joints. I Hand Surg Am. 1979;4:482-486.

14. Brüser $P$, Poss $T$, Larkin $G$. Results of proximal interphalangeal joint release for flexion contractures: midlateral versus palmar incision. J Hand Surg Am. 1999;24:288-294.

15. Houshian S, Schrøder HA. Distraction with external fixator for contractures of proximal interphalangeal joints: good outcome in 10 cases. Acta Orthop Scand. 2004;75:225-228.

16. Joshi BB. Joshi's External stabilising systembasic techniques as applied to the hand and wrist. Mumbai: JESS Research and Development Centre Publication; 1992.

17. Cziffer $E$, editor. Dynamic minifixators Budapest 1994. Literatura Medica. 